\title{
Arthroscopic removal of an osteoid osteoma of the acetabulum
}

\author{
Renske Barnhard • Eric E. J. Raven
}

Received: 10 September 2010/Accepted: 15 March 2011/Published online: 29 March 2011

(C) The Author(s) 2011. This article is published with open access at Springerlink.com

\begin{abstract}
In this case report, we describe the arthroscopic removal of an osteoid osteoma from the acetabulum in a young adolescent. After identifying the osteoid osteoma close to the cartilage with MRI and CT investigations, we decided that in this case, arthroscopic removal was the best treatment. In the case of an osteoid osteoma in the acetabulum close to the cartilage, arthroscopic removal should be considered as one can treat the associated osteochondritic lesion during this procedure.

Level of evidence IV.
\end{abstract}

Keywords Hip arthroscopy - Osteoid osteoma .

Acetabulum $\cdot$ Benign bone tumour

\section{Introduction}

An osteoid osteoma is a solitary, benign bone tumour, most commonly seen in the long bones of the lower extremities of patients in the second and third decade. Patients often present with increasing pain, pain at night with pain relief by use of nonsteroidal anti-inflammatory drugs (NSAIDs) [10]. When an osteoid osteoma is located intraarticular, it may cause swelling, joint effusion or decreased range of motion [12]. Besides the clinical characteristics, an osteoid osteoma may have a clear radiological feature, in $85 \%$ of cases, there is a small lytic nidus surrounded by reactive bone sclerosis on computer tomography (CT) [10].

R. Barnhard · E. E. J. Raven ( $\square)$ Department of Orthopaedics and Traumatology, Gelre Hospital Apeldoorn, P.O. Box 9014, 7300 DS Apeldoorn, The Netherlands e-mail: e.raven@gelre.nl
An osteoid osteoma of the acetabulum is rare $(0.5 \%)$ $[7,14]$ and difficult to diagnose. In recent literature, we found six case reports of arthroscopic removal of an osteoid osteoma of the acetabulum $[1,4,8,9]$.

\section{Case report}

A 20-year-old man visited the outpatient clinic with increasing groin pain on the right side 1 year after a highspeed traffic accident. He was not able to perform his physical demanding job anymore. Physical examination revealed a healthy young man with antalgic gait and a stiff right hip with a predominant endorotation position. Initial standard pelvic and lumbar spine radiographs were normal. An additional MRI scan showed bone oedema of the acetabulum and femoral head (Fig. 1). At this stage, the possible diagnoses were transient osteoporosis of the hip joint, osteonecrosis, stress fracture (dashboard trauma), infection or a bone tumour. The subsequent CT scan showed a small sclerotic lesion in the acetabulum. With this information, the lesion is also visible on the MRI, just underneath the cartilage (juxtaarticular). Because of this localization, the osteoid osteoma might cause a weak spot (Locus minoris resistentiae) in the cartilage and might progress to an osteochondric lesion.

Radio frequent ablation of the osteoid osteoma with close approximation cartilage was not an option, because this would not treat the osteochondric lesion. In our experience, the open surgical procedure with dislocation of the femoral head has a higher chance of complications than the arthroscopic one.

Routine hip arthroscopy was performed on the extension table. The femoral head, labrum and acetabulum were without abnormalities. A third portal was created just anterior of the anterolateral portal. We found a soft spot in 

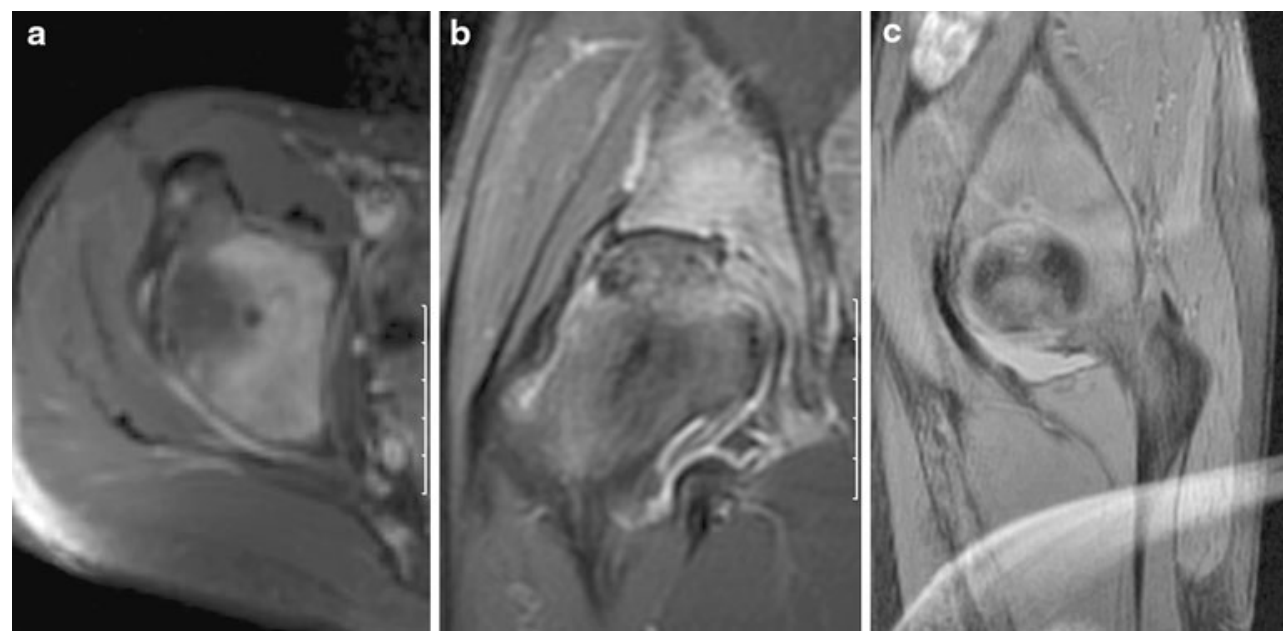

Fig. 1 MR arthrogram with gadolinium, a axial view, b coronal view, $\mathbf{c}$ sagittal view

the acetabulum just anterior of the fovea of the acetabulum. A small hook could easily be introduced into the lesion. A small loose bone fragment was removed. The cavity that remained was cleaned with curette, and micro-fracturing was performed with a chondropick.

Histological examination confirmed the diagnosis osteoid osteoma. After the operation, no further imaging was performed, due to the lack of symptoms.

\section{Discussion}

The most important finding of the present report was that an osteoid osteoma of the acetabulum can be treated by arthroscopy. Another advantage of the arthroscopy is the possibility of subsequent micro-fracturing of the remaining lesion. As shown in this case report of a juxta-articular located osteoid osteoma, presentation is often atypical and might mimic intraarticular pathology $[11,13,18]$. There is often a patient and doctors delay, sometimes even for years. Delayed treatment of a juxta-articular osteoid osteoma may cause secondary osteoarthritic changes $[12,16]$ or make the patient become medically retired at a young age. We were also on the wrong track by first requesting for an MRI scan. In our case, the CT scan eventually showed the characteristics of an osteoid osteoma, and after this, a lesion was also observed on the MRI scan. CT scan remains the modality of choice but the scan of an osteoid osteoma on a juxta-articular location may not always have such clear features as in the long bones, there may be complete absence of sclerosis. Although the MRI was not helpful in making the diagnosis, in later stage, it was of great help in locating the nidus close to the cartilage.

In recent literature, different treatment options for osteoid osteoma are described such as: open surgical excision, percutaneous CT-guided resection and CT-guided radio frequent ablation $[2,5,10,14,15,17]$. Because of the location of the osteoid osteoma close to the cartilage, we decided to perform an arthroscopy to remove the nidus and also for treatment of the overlying chondral lesion. The nidus could easily be removed, and histological examination confirmed our diagnosis. It was not possible to confirm that the nidus was fully removed. Though for adequate pain control, the full nidus has to be removed in total $[3,17]$; this could be a disadvantage of arthroscopic removal. But previous authors concluded that curettage is as effective as en bloc resection [6].

The advantages of arthroscopy are lesser surgical approach, evaluation and treatment of the cartilage defect.

Disadvantages of the technique are failure of arthroscopic approach, possibility of nerve injury and incomplete excision of the lesion or nidus. Although an MRI is not the diagnostic tool of first choice, it is helpful in describing the relation of the nidus to the articular cartilage.

Open Access This article is distributed under the terms of the Creative Commons Attribution Noncommercial License which permits any noncommercial use, distribution, and reproduction in any medium, provided the original author(s) and source are credited.

\section{References}

1. Alvarez MS, Moneo PR, Palacios JA (2001) Arthroscopic extirpation of an osteoid osteoma of the acetabulum. Arthroscopy 17:768-771

2. Callaghan JJ, Salvati EA, Pellicci PM, Bansal M, Ghelman B (1988) Evaluation of benign acetabular lesions with excision through the Ludloff approach. Clin Orthop Relat Res 237: 170-178

3. Carter TR (1990) Osteoid osteoma of the hip: an alternate method of excision. Orthop Rev 19:903-905

4. Chang BK, Ha YC, Lee YK, Hwang DS, Koo KH (2010) Arthroscopic excision of osteoid osteoma in the posteroinferior portion of the acetabulum. Knee Surg Sports Traumatol Arthrosc 18:1685-1687 
5. Fenichel I, Garniack A, Morag B, Palti R, Salai M (2006) Percutaneous CT-guided curettage of osteoid osteoma with histological confirmation: a retrospective study and review of the literature. Int Orthop 30:139-142

6. Gunes T, Erdem M, Bostan B, Sen C, Sahin SA (2008) Arthroscopic excision of the osteoid osteoma at the distal femur. Knee Surg Sports Traumatol Arthrosc 16:90-93

7. Karray S, Zlitni M, Karray M, Moalla M, Zouari M, Douik M, Sliman N (1993) Osteoid osteoma of the acetabulum. Int Orthop 17:54-56

8. Khapchik V, O’Donnell RJ, Glick JM (2001) Arthroscopically assisted excision of osteoid osteoma involving the hip. Arthroscopy $17: 56-61$

9. Lee DH, Malawer MM (1992) Staging and treatment of primary and persistent (recurrent) osteoid osteoma. Evaluation of intraoperative nuclear scanning, tetracycline fluorescence, and tomography. Clin Orthop Relat Res 281:229-238

10. Lee EH, Shafi M, Hui JH (2006) Osteoid osteoma: a current review. J Pediatr Orthop 26:695-700

11. Mitsui Y, Gotoh M, Yoshida T, Hirai Y, Shinozaki T, Nakama K, Higuchi F, Nagata K (2008) Osteoid osteoma of the proximal humerus: a misleading case. J Shoulder Elbow Surg 17:e13-e15
12. Mounach A, Nouijai A, Ghozlani I, Ghazi M, Achemlal L, Bezza A, El Maghraoui A (2008) Osteoid osteoma of the acetabulum: a case report. Rheumatol Int 28:601-603

13. Nourissat G, Kakuda C, Dumontier C (2007) Arthroscopic excision of osteoid osteoma of the elbow. Arthroscopy 23(799): e791-e794

14. Parlier-Cuau C, Nizard R, Champsaur P, Hamze B, Quillard A, Laredo JD (1999) Osteoid osteoma of the acetabulum. Three cases treated by percutaneous resection. Clin Orthop Relat Res 365:167-174

15. Pratali R, Zuiani G, Inada M, Hanasilo C, Reganin L, Etchebehere E, Etchebehere M (2009) Open resection of osteoid osteoma guided by a gamma-probe. Int Orthop 33:219-223

16. Szendroi M, Kollo K, Antal I, Lakatos J, Szoke G (2004) Intraarticular osteoid osteoma: clinical features, imaging results, and comparison with extraarticular localization. J Rheumatol 31: 957-964

17. Witt J (2002) Management of osteoid osteoma. Hosp Med 63:207-209

18. Zupanc O, Sarabon N, Strazar K (2007) Arthroscopic removal of juxtaarticular osteoid osteoma of the elbow. Knee Surg Sports Traumatol Arthrosc 15:1240-1243 